\title{
Intestinal Stem Cells: Common Signal Pathways, Human Disease Correlation, and Implications for Therapies
}

\author{
Jignesh Dalal and Mohamed Radhi \\ Division of Hematology, Oncology, and Stem Cell Transplantation, Children's Mercy Hospital, 2401 Gillham Road, Kansas City, \\ MO 64108, USA \\ Correspondence should be addressed to Mohamed Radhi; maradhi@cmh.edu
}

Received 25 October 2012; Accepted 15 November 2012

Academic Editors: A. Chapel, F. Fagioli, I. E. Hoefer, B. Machalinski, and B. Rogister

Copyright (C) 2013 J. Dalal and M. Radhi. This is an open access article distributed under the Creative Commons Attribution License, which permits unrestricted use, distribution, and reproduction in any medium, provided the original work is properly cited.

Enterocytes originating from gastrointestinal stem cells are basic building blocks of villi and crypts in human intestine. Little is known about intestinal stem cells (ISCs), their interaction with niche, and key pathways in their regulation. In this paper, we have reviewed the characteristics of ISC, its interaction with niche, and the understanding of key signaling pathways like Wnt. A better understanding of all of this will help to better utilize novel therapies like mesenchymal stromal cells (MSCs), R-spondin1, and sulindac in various disorders like colon cancer, graft-versus-host disease, intestinal polyposis, and radiation-related bowel injuries.

\section{Introduction}

The intestinal epithelium is composed of five primary cell types: the common absorptive enterocyte, the enteroendocrine cell, the mucous-secreting goblet cell, the tuft cell, and the Paneth cells $[1,2]$. The epithelium of mammalian intestine undergoes rapid turnover to be replenished every 3-5 days [3]. Proliferation occurs near the base of the crypts, with cell maturation occurring as they migrate along the crypt/villus axis. The crypt is the monoclonal proliferative compartment of the intestinal epithelium. The villus represents the differentiated polyclonal compartment, as its cells derive from several crypts. Potten and his colleagues showed in an adult mouse model that every villus has approximately 7000 cells in the duodenum and each villus is surrounded by approx 15 crypts containing 350-400 cells. There are approximately one million crypts in the human small intestine [4]. Enterocytes are the most common epithelial cells and are responsible for absorption of nutrients and water via active and passive transport. Goblet cells secrete mucins and other proteins that are used for lubrication and as a barrier defense against pathogens, and Paneth cells secrete lysozyme to prevent bacterial infection $[5,6]$. Driven by the Notch signaling pathway, enteroendocrine cells having ten different subtypes comprise $1 \%$ of total epithelium. They are responsible for secreting a variety of hormones that participate in glucose homeostasis [7], satiety (ghrelin), pH balance (secretin), gall bladder contraction (cholecystokinin), gut motility (neurotensin and motilin), and the regulation of pancreatic and pituitary hormone secretion (somatostatin) [8-10].

\section{Intestinal Stem Cells (ISCs)}

Maintenance of the functional integrity of intestinal epithelium is a challenging task which is done by small numbers of long-lived multipotent cells called intestinal stem cells (ISCs) [11]. The discovery of the ISC remained elusive till early in the 21 st century because of the lack of molecular markers and the difficulty of culturing. Potten and his colleagues inferred its localization at crypt bases by demonstrating retention of DNA-labeling agents at position 4 (4 cells above the crypt base) for many weeks in the irradiated mouse model [12]. Cheng and his colleagues in 2006 showed that some proliferative cells, called crypt base columnar cells (CBCs), residing below the +4 position and intermingled with Paneth cells, had features consistent with those of ISC [13]. In 2007, Barker et al. discovered $\operatorname{Lgr} 5$, a gene encoding a G proteincoupled receptor, whose expression was restricted to CBCs as genetic marker of ISC in Cre recombinase mouse model [14]. 
These mice harbor heterozygous Lgr5-EGFP-IRES-creERT2 "knock-in" allele that abolishes Lgr5 gene function and expresses EGFP and CreERT2 fusion proteins from the Lgr5 promoter/enhancer elements. EGFP fluorescence is observed in crypt base columnar cells in small intestine (a.k.a. stem cells of the small intestine) and colon. Cre-ERT2 fusion gene activity is inducible; it is observed in the same cells only following tamoxifen administration. In the same paper, they also made clear that +4 cells are distinct from CBCs in view of low-dose radiation (less than 1 gray) sensitivity. In 2008, Sangiorgi and Capecchi linked another marker called Bmil (chromatin remodeling protein) to ISCs, similar to hematopoetic and neural stem cells. They could demonstrate, in similar Cre recombinase mouse model, expression of Bmil in long-lived cell clones of different intestinal lineages. Ablation of Bmil by targeted expression of diphtheria toxin resulted in depletion of the entire epithelium [15]. It is likely that Bmil and Lgr5 may not represent the same population of cells, as Lgr5 is expressed throughout the gastrointestinal tract including stomach, small intestine, and colon crypts, whereas Bmil expression is restricted to the most proximal half of the small intestine. Due to cell cycle differences, crypt Bmil + cells generate progeny with relatively slow kinetics compared to Lgr5+ ISCs. In 2009, two different groups were able to successfully propagate and maintain ISC in longterm culture $[16,17]$. Sato et al. used laminin-rich Matrigel and growth medium containing the Wnt agonist R-spondin, epidermal growth factor, and Bmp antagonist Noggin. They were able to create organoids, comprising more than $40 \mathrm{crypt}$ domains surrounding a central lumen lined by a villus-like epithelium, and organoids were cultured for more than 8 months without losing their characteristics [16]. Ootani et al. used 3D matrix of type I collagen gel under an air-liquid interface for sustained in vitro intestinal epithelial culture of Wnt-dependent stem cells [17].

\section{Stem Cell Niche}

The intestinal crypt-niche interaction is important in the function, maintenance, and proliferation of progenitor stem cells found at the bases of intestinal crypts. The cells in the ISC niche consist of microvascular endothelial cells, mesenchyme-derived intestinal subepithelial myofibroblasts (ISEMFs), pericryptal macrophages, enteric neurons, intraepithelial lymphocytes (IELs), and microbes [18]. ISEMFs are located in the lamina propria in close proximity to the crypt cells and migrate upward from the crypt base to the villous tip along with maturing epithelial cells, establishing signaling crosstalk and regulating epithelial cell differentiation $[18,19]$. ISEMFs have qualities of both smooth muscle cells and fibroblasts and interact via various conserved intracellular pathways such as Wnt, Bmp, and Notch. They exert their function on neighboring epithelial cells via both direct contact and paracrine modalities [19, 20]. ISEMFs also interact with pericryptal macrophages with subsequent release of PGE2 that could reduce apoptosis of enterocytes [20]. Pericryptal macrophages form synapses with crypt stem cells and secrete growth factors to stimulate ISC proliferation upon activation of Toll-like receptors sensing the entry of bacteria and other intestinal pathogens [21]. Like ISEMFs, Paneth cells have also been implicated in the regulation of proliferation and differentiation of ISCs [22].

The functional role of the ISC niche is very well demonstrated by the protective role of autologous BM stromal cell transplant (BMASCT) after lethal high-dose abdominal radiation (12-20 Gy) [23]. The authors of this study observed that BMASCT restores the ISC niche including the pericryptal macrophages, endothelial, cells and ISEMF, leading to better restoration of the function and architecture of intestinal villi [23].

\section{Signal Transduction Pathways for Regeneration and Malignancy}

Wnt signaling plays a very important role in the regulation of intestinal epithelial cell proliferation during their maturation or regeneration [24]. Wnt proteins form a family of highly conserved secreted signaling molecules that regulate cell-tocell interactions during embryogenesis. Wnt signals are transduced through two pathways, canonical and noncanonical, determining cell fate and cell motility, respectively [19]. Wnt proteins bind to receptors of the Frizzled and LRP families on the cell surface. Through several cytoplasmic relay components, the signal is transduced to beta-catenin, which enters the nucleus to activate transcription of Wnt target genes transgenic expression of the secreted Wnt inhibitor Dickkopf1 (Dkk-1) leading to reduced small intestinal epithelial proliferation and loss of crypts [25]. Conversely, injection of R-spondin, a Wnt agonist which protects against ISC damage, enhances restoration of injured intestinal epithelium and inhibits subsequent inflammatory cytokine cascades [26]. Upregulation of Wnt family ligands and downregulation of Wnt inhibitors occur during the early stage of carcinogenesis mediated by chronic inflammation [27]. Similarly, $\beta$-catenin and APC proteins, which are components of the Wnt/ $\beta$ catenin signaling canonical pathway, play a critical role in oncogenesis of colorectal cancer [24].

\section{Human Diseases Correlation}

In the bowel, the mucosal stem cells in the base of the colonic crypts may accumulate mutations and hence lead to tumor development. These stem cells are characterized by their capacities for long life and self-renewal. Normal colonic stem cells exhibit an incredible rate of cell production and turnover in order to maintain the colonic mucosa. Cancer may therefore develop as a result of alteration of this process through the accumulation of mutations and damage within the controlling stem cells. Methods of investigating gastrointestinal stem cells (GSCs) in the human have proved to be difficult. The first studies of clonality in the gastrointestinal mucosa in humans utilized the human colorectal carcinoma cell line HRA19, which produces single-cell clones that can be grown as polarized monolayers in vitro. When engrafted subcutaneously into nude mice, these clones produce tumors 
that are histologically identical to the original tumor, containing columnar, goblet, and neuroendocrine cells. Thus, these malignant epithelial cells are multipotential and can produce every differentiated cell type in the human colorectal epithelia [7]. The malignant counterpart of the normal ISC is characterized by being CD133+ enriched, which can significantly increase the capability of these cells to initiate tumor growth $[28,29]$. In humans and mice with APC gene mutations, the loss of the APC tumor suppressor leads to increased expression of $\beta$-catenin, resulting in cellular "proliferation and resistance to apoptosis" [28-31]. Though of various nature, these APC mutations will result in stabilizing $\beta$-catenin and the perpetual activation of the Wnt program whether or not an extracellular signal is present [32]. LGR5, an intestinal stem cell marker that is a Wnt target, may have a role in each stage of colorectal cancer (CRC) stage and lead to cancer progression. This also appears to be true for another precancerous condition, Barrett's Esophagus, suggesting that it will likely serve as a useful cancer stem cell marker $[33,34]$. While increased Wnt/ $\beta$-catenin pathway signaling leads to an increase in the proliferative capacity of cancer stem cells, the BMP pathway inhibits their proliferation [35]. He et al. showed that BMP signaling suppresses Wnt signaling to ensure a balanced control of stem cell self-renewal. Thus, BMP signaling may control the duplication of intestinal stem cells, suggesting the possibilities of new drug development for the control of colon cancer. A similar role of GSC in gastric cancer has been observed. Patients with chronic atrophic gastritis, when infested by Helicobacter pylori, are prone to gastric cancer, indicating that proliferating and nonproliferating GSCs provide a habitat for this bacteria that can lead to increased risk of cancer development [36].

In conclusion, increasing evidence supports the presence of a cancer stem cell as the cause of gastrointestinal tumor establishment, progression, relapse, and metastasis. Identification of the origin of the GSC remains elusive in human CRC; however, progress is being made in mouse models of intestinal cancer. The precise role of the GSC in these tumorigenic steps of CRC also remains unclear. Additionally, the interaction of colorectal GSC with the cellular microenvironment, both at the site of tumor initiation and at sites of metastatic deposit, must be further investigated. This is particularly needed given the importance of the microenvironmental niche in the function and maintenance of somatic stem cells.

\section{Therapeutic Application}

The intestinal mucosa has an amazing proliferative capacity, enabling normal homeostasis as well as rapid restoration of its physiological functions following injury. The ability to do this resides with the epithelial stem cells. Recent advances toward the isolation and characterization of epithelial stem cells have paved the way for exploring novel therapeutic approaches for gastrointestinal disease. Possible stem cell-based therapy of gastrointestinal disorders ranges from the repair of damaged mucosa to the engineering of artificial intestinal constructs for patients with short bowel syndrome. As mentioned before, aberrant activation of $\mathrm{Wnt} / \beta$-catenin signaling is observed in many human cancers, with genetic mutations of Wnt signaling pathway components being primarily responsible for this aberrant activation. Dysregulation of different components of this pathway can lead to different types of gastrointestinal cancer [37]. Inhibitors of this complex and large Wnt/ $\beta$-catenin signaling pathway have been developed for the treatment of cancers. These fall generally into two broad categories: biological inhibitors, such as monoclonal antibodies, recombinant small inhibitory nucleic acids (siNAs), and other small molecules such as NSAIDs, and imatinib [38]. In a very elegant group of experiments in a mouse model, Qiu et al. [39] recently delineated the mechanism by which NSAIDs can prevent colon cancer at the cellular level. They found that dietary sulindac removed intestinal stem cells with $\beta$-Catenin accumulation, suppressed $\beta$-Catenin phosphorylation, and induced apoptosis in intestinal stem cells of $\mathrm{APC}^{\mathrm{Min} /+}$ mice. NSAIDs also induced apoptosis in human colonic polyps and removed cells with aberrant Wnt signaling. While Wnt/ $\beta$ catenin signaling pathway antagonists and inhibitors can be used to stop the growth and spread of cancer, agonists of this pathway have been shown to promote gut healing. Takashima et al. [26] have recently shown that the Wnt signaling pathway agonist R-spondin1 (R-Spo1) protected against ISC damage, enhanced restoration of injured intestinal epithelium, and inhibited subsequent inflammatory cytokine cascades in mismatched murine bone marrow transplant recipients.

While the use of this approach helped gut damage healing by promoting the rapid growth of the ISCs, Van't Land et al. [40] have shown earlier in a rat model for induced small intestinal damage that oral supplementation of rats exposed to methotrexate with a Transforming Growth Factor- $\beta 2$ enriched fraction prepared from bovine milk by reversibly arresting growth of epithelial stem cells can protect small intestinal epithelial cells against cell-cycle-specific chemotherapeutic agents by arresting the cells in G1-phase. Administration of TGF- $\beta 2$ has also been shown to reduce the chemotherapy-associated weight loss and ileal villous atrophy by reducing cell proliferation in the normal stem cell population.

In a very interesting publication, Lam et al. [41] described in detail the mechanisms by which a four-herb Chinese medicine formula reduces chemotherapy-induced gastrointestinal toxicity. This formula acts at the level of the gastrointestinal progenitor and stem cells. The herbal compound PHY906 appeared to be responsible. It induced the expression of the stem cell markers CD44, Lgr5, Ascl2, and Olfm4 and increased the expression of Wnt signaling components 4 days after CPT-11 chemotherapy. These results suggest that PHY906 may promote progenitor cell regeneration after CPT-11 treatment by stimulating Wnt signaling.

\section{Cellular Therapy}

Multipotent mesenchymal stromal cells (MSCs) are a rare population of cells possessing stem cell characteristics. Evidence is mounting that such multipotent progenitors may reside within the perivascular niche of several organs, and 
they are candidates for cellular therapy in different settings, ranging from inflammatory bowel disease (IBD) to graftversus-host disease (GVHD). MSCs can be derived from a variety of tissues including the bone marrow $[42,43]$. Recently, Lanzoni et al. [44] were able to isolate a population of MSCs from intestinal tissue. This population of cells was extensively expandable, showed typical markers of this cell type (CD29+, CD44+, CD73+, CD105+ and CD166+, CD14-, and CD34- and CD45-), and were able to induce the differentiation and organization of intestinal epithelial cells. The isolation of these stem cells may open new possibilities for therapeutic enhancement of intestinal tissue regeneration.

MSCs do not have to be strictly originating from the intestine to be considered for intestinal tissue engineering or cellular therapy. In a very interesting tissue engineering model by Sîrbu-Boițe et al. [45], MSCs obtained from human and rat bone marrows were cultured and seeded into a collagen scaffold. Three-dimentional patches were sutured in areas of the rat gut and have shown to integrate with the rest of the gut and promote gut functionality and healing. Even more simply, researchers have also shown that local injection of these cells in damaged gut can directly induce healing. Hayashi et al. [46] showed that topical implantation of MSCs freshly isolated from rat bone marrow into a model of colitis significantly accelerated healing of the damaged mucosa compared with vehicle-injected controls. The MSCs expressed vascular endothelial growth factor (VEGF) and transforming growth factor (TGF)- $\beta 1$ in vitro and after the implantation. Other researchers have also shown that locally transplanted bone-marrow-derived MSC improved ischemic colonic anastomotic sites in animal models [47]. Bone-marrow-derived MSCs may not be the only type of cells in the bone marrow that can help repair damaged intestinal epithelia. Okamoto et al. [48] reported their discovery of a bone-marrow-derived epithelial cell that promoted the regeneration of damaged epithelia in the human intestinal tract. These cells were donor-derived after allogeneic bone marrow transplant and have persisted for years. They proliferate following intestinal epithelial injury by GVHD. Precise analysis of epithelial cell lineages revealed that, during epithelial regeneration, secretory lineage epithelial cells that originated from bone marrow significantly increased in number. Their conclusion was that bone marrow may serve as a useful source for intestinal epithelial cell precursors. However, the precise role of the bone marrow derived MSC in IBD and their interaction with the gastrointestinal tract are not completely settled or well understood. Nemoto et al. [42] showed very nicely in an animal model that IL-7 produced by MSC from IL-7 ${ }^{-/-}$X RAG-1 ${ }^{-/-}$mice transplanted with IL-7 was sufficient, and BM MSC expressed IL-7 only in the $\mathrm{BM}$ and not in the colon and lead to the development of IBD and colitis when injected with $\mathrm{CD}^{+} \mathrm{CD} 45 \mathrm{RB}^{\text {high }}$. This recent and elegant work has shed a new light on the dynamic and complex interaction between the BM-derived MSCs and IBD. This work comes to validate what clinicians have observed in a clinical model few years ago. Dean et al. [49] evaluated the association of serum IL-7 levels in 31 patients who received reduced intensity allogeneic stem cell transplant. Of the 31 patients, 14 patients developed acute graft-versus-host disease (GVHD) (of any site, gut, liver, or skin). Almost 60\% (8/14) developed gut acute GVHD. Acute GVHD correlated significantly with higher IL-7 levels on day +7 and day +14 after transplant. IL-7 levels at day +14 also correlated with the severity of acute GVHD. So, if we try to connect the BM-derived MSC puzzle together, they seem to have a complex and dynamic role in both inciting and healing IBD. While on the cellular level they can participate by themselves in local tissue regeneration of the damaged gut and repair of the IBD inflammation, they can also have an inciting role leading to more cytokine-driven inflammation and IBD and IBD-like disease in the form of GVHD. The final participation and the role they play can be a balance of both directions and seem to be dependant on a multitude of other factors.

\section{Summary}

In summary, identification of ISCs, and an understanding of niche and signaling pathways have lead to a better understanding of various diseases like GI cancers, polyp formation, radiation and chemotherapy-induced intestinal damage, inflammatory bowel diseases, and so forth. A better understanding has also lead to the development of refined animal disease model to test new drugs and biologic and cellular therapies for malignant and nonmalignant gastrointestinal disorders paving the way for novel therapies for mankind.

\section{Authors' Contribution}

Both authors contributed equally to this paper.

\section{References}

[1] H. Cheng and C. P. Leblond, "Origin, differentiation and renewal of the four main epithelial cell types in the mouse small intestine. V. Unitarian theory of the origin of the four epithelial cell types," American Journal of Anatomy, vol. 141, no. 4, pp. 537-561, 1974.

[2] F. Gerbe, J. H. van Es, L. Makrini et al., "Distinct ATOH1 and Neurog3 requirements define tuft cells as a new secretory cell type in the intestinal epithelium," Journal of Cell Biology, vol. 192, no. 5, pp. 767-780, 2011.

[3] A. Shaker and D. C. Rubin, "Intestinal stem cells and epithelialmesenchymal interactions in the crypt and stem cell niche," Translational Research, vol. 156, no. 3, pp. 180-187, 2010.

[4] C. S. Potten and M. Loeffler, "Stem cells: attributes, cycles, spirals, pitfalls and uncertainties. Lessons for and from the crypt," Development, vol. 110, no. 4, pp. 1001-1020, 1990.

[5] P. Dharmani, V. Srivastava, V. Kissoon-Singh, and K. Chadee, "Role of intestinal mucins in innate host defense mechanisms against pathogens," Journal of Innate Immunity, vol. 1, no. 2, pp. 123-135, 2009.

[6] A. deFoneska and J. D. Kaunitz, "Gastroduodenal mucosal defense," Current Opinion in Gastroenterology, vol. 26, no. 6, pp. 604-610, 2010.

[7] M. Brittan and N. A. Wright, "Gastrointestinal stem cells," Journal of Pathology, vol. 197, no. 4, pp. 492-509, 2002. 
[8] S. Efendic and N. Portwood, "Overview of incretin hormones," Hormone and Metabolic Research, vol. 36, no. 11-12, pp. 742-746, 2004.

[9] C. S. Lee and K. H. Kaestner, "Clinical endocrinology and metabolism. Development of gut endocrine cells," Best Practice and Research: Clinical Endocrinology and Metabolism, vol. 18, no. 4, pp. 453-462, 2004.

[10] G. Rindi, A. B. Leiter, A. S. Kopin, C. Bordi, and E. Solcia, "The "normal" endocrine cell of the gut: changing concepts and new evidences," Annals of the New York Academy of Sciences, vol. 1014, pp. 1-12, 2004.

[11] E. Batlle, "A new identity for the elusive intestinal stem cell," Nature Genetics, vol. 40, no. 7, pp. 818-819, 2008.

[12] C. S. Potten, G. Owen, and D. Booth, "Intestinal stem cells protect their genome by selective segregation of template DNA strands," Journal of Cell Science, vol. 115, part 11, pp. 2381-2388, 2002.

[13] M. Bjerknes and H. Cheng, "Intestinal epithelial stem cells and progenitors," Methods in Enzymology, vol. 419, pp. 337-383, 2006.

[14] N. Barker, J. H. van Es, J. Kuipers et al., "Identification of stem cells in small intestine and colon by marker gene Lgr5," Nature, vol. 449, no. 7165, pp. 1003-1007, 2007.

[15] E. Sangiorgi and M. R. Capecchi, "Bmil is expressed in vivo in intestinal stem cells," Nature Genetics, vol. 40, no. 7, pp. 915-920, 2008.

[16] T. Sato, R. G. Vries, H. J. Snippert et al., "Single Lgr5 stem cells build crypt-villus structures in vitro without a mesenchymal niche," Nature, vol. 459, no. 7244, pp. 262-265, 2009.

[17] A. Ootani, X. Li, E. Sangiorgi et al., "Sustained in vitro intestinal epithelial culture within a Wnt-dependent stem cell niche," Nature Medicine, vol. 15, no. 6, pp. 701-706, 2009.

[18] T. H. Yen and N. A. Wright, "The gastrointestinal tract stem cell niche," Stem Cell Reviews, vol. 2, no. 3, pp. 203-212, 2006.

[19] M. Katoh, "WNT signaling in stem cell biology and regenerative medicine," Current Drug Targets, vol. 9, no. 7, pp. 565-570, 2008.

[20] T. M. Yeung, L. A. Chia, C. M. Kosinski, and C. J. Kuo, "Regulation of self-renewal and differentiation by the intestinal stem cell niche," Cellular and Molecular Life Sciences, vol. 68, no. 15, pp. 2513-2523, 2011.

[21] S. Samuel, R. Walsh, J. Webb, A. Robins, C. Potten, and Y. R. Mahida, "Characterization of putative stem cells in isolated human colonic crypt epithelial cells and their interactions with myofibroblasts," American Journal of Physiology, vol. 296, no. 2, pp. C296-C305, 2009.

[22] T. Sato, J. H. van Es, H. J. Snippert et al., "Paneth cells constitute the niche for Lgr5 stem cells in intestinal crypts," Nature, vol. 469, no. 7330, pp. 415-418, 2011.

[23] S. Saha, P. Bhanja, R. Kabarriti, L. Liu, A. A. Alfieri, and C. Guha, "Bone marrow stromal cell transplantation mitigates radiationinduced gastrointestinal syndrome in mice," PLoS One, vol. 6, no. 9, article e24072, 2011.

[24] D. Pinto and H. Clevers, "Wnt, stem cells and cancer in the intestine," Biology of the Cell, vol. 97, no. 3, pp. 185-196, 2005.

[25] D. Pinto, A. Gregorieff, H. Begthel, and H. Clevers, "Canonical Wnt signals are essential for homeostasis of the intestinal epithelium," Genes and Development, vol. 17, no. 14, pp. 1709-1713, 2003.

[26] S. Takashima, M. Kadowaki, K. Aoyama et al., "The Wnt agonist R-spondin 1 regulates systemic graft-versus-host disease by protecting intestinal stem cells," Journal of Experimental Medicine, vol. 208, no. 2, pp. 285-294, 2011.
[27] M. Katoh and M. Katoh, "WNT signaling pathway and stem cell signaling network," Clinical Cancer Research, vol. 13, no. 14, pp. 4042-4045, 2007.

[28] P. Salama and C. Platell, "Colorectal cancer stem cells," ANZ Journal of Surgery, vol. 79, no. 10, pp. 697-702, 2009.

[29] L. G. van der Flier and H. Clevers, "Stem cells, self-renewal, and differentiation in the intestinal epithelium," Annual Review of Physiology, vol. 71, pp. 241-260, 2009.

[30] K. P. Janssen, P. Alberici, H. Fsihi et al., "APC and oncogenic KRAS are synergistic in enhancing Wnt signaling in intestinal tumor formation and progression," Gastroenterology, vol. 131, no. 4, pp. 1096-1109, 2006.

[31] S. Miyamoto and D. W. Rosenberg, "Role of Notch signaling in colon homeostasis and carcinogenesis," Cancer Science, vol. 102, no. 11, pp. 1938-1942, 2011.

[32] F. Melo, L. Vermeulen, D. Richel, and J. P. Medema, "Targeting Wnt signaling in colon cancer stem cells," Clinical Cancer Research, vol. 17, no. 4, pp. 647-653, 2011.

[33] L. Becker, Q. Huang, and H. Mashimo, "Lgr5, an intestinal stem cell marker, is abnormally expressed in Barrett's esophagus and esophageal adenocarcinoma," Diseases of the Esophagus, vol. 23, no. 2, pp. 168-174, 2010.

[34] K. Takeda, I. Kinoshita, Y. Shimizu, Y. Matsuno, T. Shichinohe, and H. Dosaka-Akita, "Expression of LGR5, an intestinal stem cell marker, during each stage of colorectal tumorigenesis," Anticancer Research, vol. 31, no. 1, pp. 263-270, 2011.

[35] J. P. Medema and L. Vermeulen, "Microenvironmental regulation of stem cells in intestinal homeostasis and cancer," Nature, vol. 474, no. 7351, pp. 318-326, 2011.

[36] X. C. He, J. Zhang, W. G. Tong et al., "BMP signaling inhibits intestinal stem cell self-renewal through suppression of Wnt$\beta$-catenin signaling," Nature Genetics, vol. 36, no. 10, pp. 1117-1121, 2004.

[37] J. D. Oh, H. Kling-Bäckhed, M. Giannakis, L. G. Engstrand, and J. I. Gordon, "Interactions between gastric epithelial stem cells and Helicobacter pylori in the setting of chronic atrophic gastritis," Current Opinion in Microbiology, vol. 9, no. 1, pp. 21-27, 2006.

[38] H. Yao, E. Ashihara, and T. Maekawa, "Targeting the Wnt/ $\beta$ catenin signaling pathway in human cancers," Expert Opinion on Therapeutic Targets, vol. 15, no. 7, pp. 873-887, 2011.

[39] W. Qiu, X. Wang, B. Leibowitz et al., "Chemoprevention by nonsteroidal anti-inflammatory drugs eliminates oncogenic intestinal stem cells via SMAC-dependent apoptosis," Proceedings of the National Academy of Sciences of the United States of America, vol. 107, no. 46, pp. 20027-20032, 2010.

[40] B. Van't Land, H. P. Meijer, J. Frerichs et al., “Transforming growth factor- $\beta 2$ protects the small intestine during methotrexate treatment in rats possibly by reducing stem cell cycling," British Journal of Cancer, vol. 87, no. 1, pp. 113-118, 2002.

[41] W. Lam, S. Bussom, F. Guan et al., "The four-herb Chinese medicine PHY906 reduces chemotherapy-induced gastrointestinal toxicity," Science Translational Medicine, vol. 2, no. 45, p. 45ra59, 2010.

[42] Y. Nemoto, T. Kanai, M. Takahara et al., "Bone marrowmesenchymal stem cells are a major source of interleukin-7 and sustain colitis by forming the niche for colitogenic CD4 memory T cells," Gut. In press.

[43] A. Milanesi, J.-W. Lee, Z. Li et al., "b-cell regeneration mediated by human bone marrow mesenchymal stem cells," PLoS One, vol. 7, no. 8, article e42177, 2012. 
[44] G. Lanzoni, F. Alviano, C. Marchionni et al., "Isolation of stem cell populations with trophic and immunoregulatory functions from human intestinal tissues: potential for cell therapy in inflammatory bowel disease," Cytotherapy, vol. 11, no. 8, pp. 1020-1031, 2009.

[45] M. P. Sîrbu-Boeți, M. Chivu, L. L. Pâslaru et al., "Transplantation of mesenchymal stem cells cultured on biomatrix support induces repairing of digestive tract defects, in animal model," Chirurgia, vol. 104, no. 1, pp. 55-65, 2009.

[46] Y. Hayashi, S. Tsuji, M. Tsujii et al., "Topical implantation of mesenchymal stem cells has beneficial effects on healing of experimental colitis in rats," Journal of Pharmacology and Experimental Therapeutics, vol. 326, no. 2, pp. 523-531, 2008.

[47] G. Adas, S. Arikan, O. Karatepe et al., "Mesenchymal stem cells improve the healing of ischemic colonic anastomoses (experimental study)," Langenbeck's Archives of Surgery, vol. 396, no. 1, pp. 115-126, 2011.

[48] R. Okamoto, T. Matsumoto, and M. Watanabe, "Regeneration of the intestinal epithelia: regulation of bone marrow-derived epithelial cell differentiation towards secretory lineage cells," Human Cell, vol. 19, no. 2, pp. 71-75, 2006.

[49] R. M. Dean, T. Fry, C. Mackall et al., "Association of serum interleukin-7 levels with the development of acute graft-versushost disease," Journal of Clinical Oncology, vol. 26, no. 35, pp. 5735-5741, 2008. 


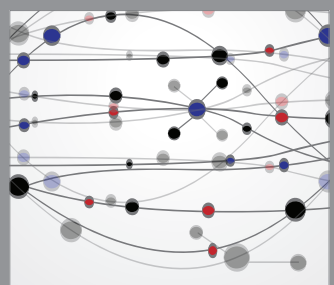

The Scientific World Journal
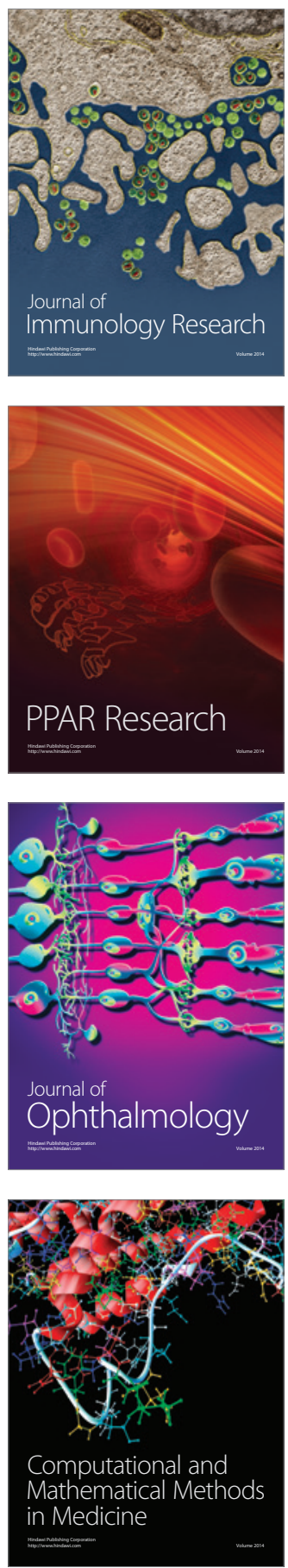

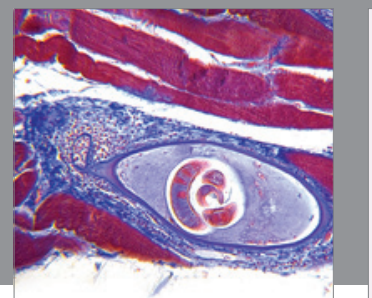

Gastroenterology

Research and Practice
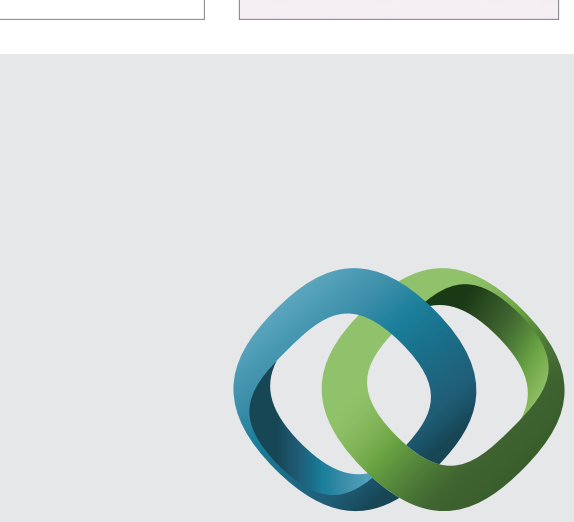

\section{Hindawi}

Submit your manuscripts at

http://www.hindawi.com
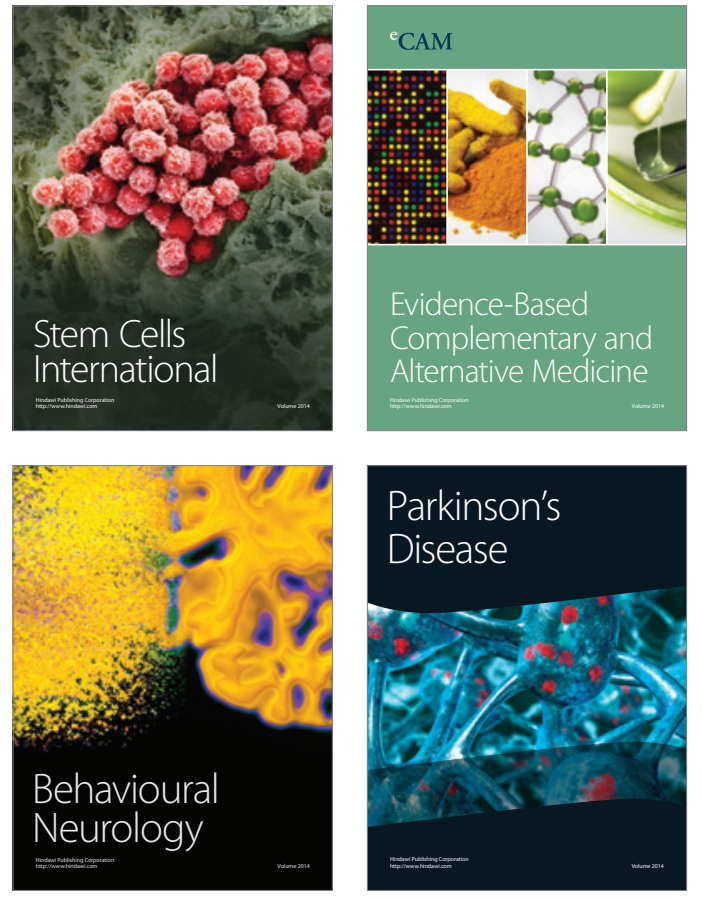
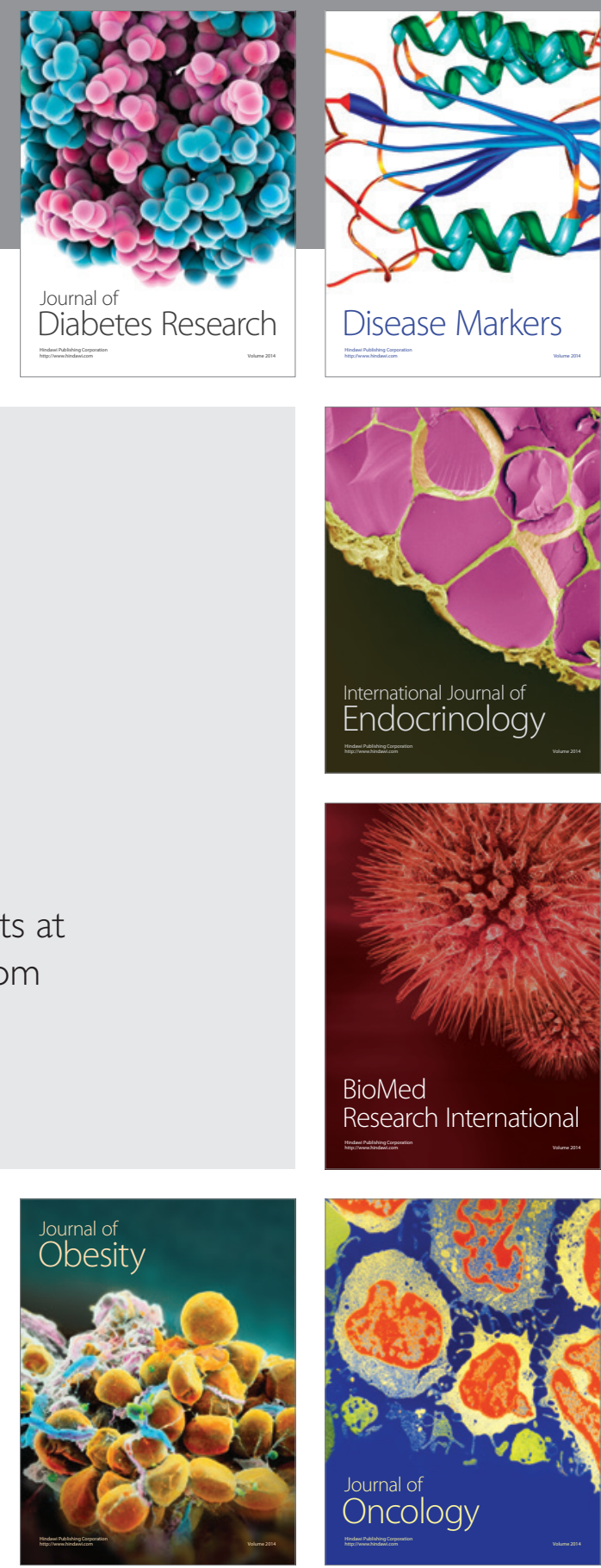

Disease Markers
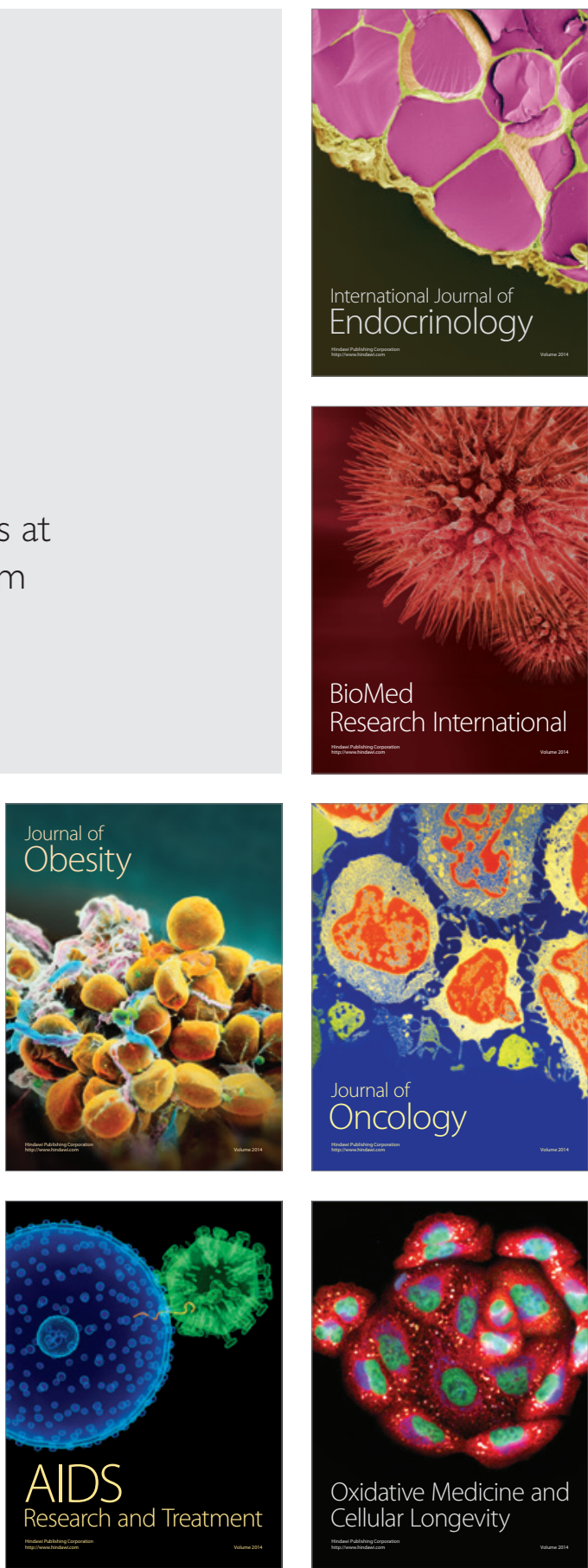